\title{
Richtig ausgerüstet für den Notfall?
}

\author{
Immer und überall können sich gefahrdrohende Notfälle ereignen. So treten sie auch während der oft \\ unkomplizierten Routineeingriffe in der Zahnarztpraxis auf. Wie sollte eine Zahnarztpraxis aus juristischer \\ Sicht für diesen Fall ausgestattet sein? Rechtsanwältin Katharina Wandtke gibt Auskunft.
}

\begin{abstract}
djz: Frau Wandtke, was ist dran an der Behauptung, den Zahnärzten werde von "offizieller" Seite immer wieder gesagt, sie benötigen in ihren Praxen einen Verbandskasten nach DIN 13157, einen Notfallkoffer hingegen benötigen sie nicht.
\end{abstract}

Katharina Wandtke: Das kann man nicht in einen Topf werfen. In Deutschland sind Verbandskästen in den Bereichen Straßenverkehr und Arbeitsschutz vorgeschrieben. Als Betriebsstätte anderer Art genügt in der Zahnarztpraxis, anders als auf einer Baustelle oder einem Industriebetrieb, ein kleiner Verbandskasten nach DIN 13157. Falls sich ein Mitarbeiter beim Kaffeekochen verbrüht, sich die Finger einklemmt oder ähnliches passiert. Die Frage, ob ein Notfallkoffer vorgehalten werden sollte, steht in einem ganz anderen Zusammenhang, nämlich mit der Behandlung von Patienten, und hängt von dem aktuell geltenden zahnmedizinischen Behandlungsstandard ab.

djz: Gibt es ein Gesetz, das den Zahnarzt verpflichtet, einen Notfallkoffer vorzuhalten?

Wandtke: Ja, das gibt es. Die Haftung des Arztes resultiert aus der sowohl zivilrechtlich als auch strafrechtlich normierten besonderen Verantwortlichkeit. Haftungsbegründend ist jeweils der Verstoß gegen den geltenden Sorgfaltsmaßstab, etwa ein Verfehlen dessen, was in seinem Fachbereich als Stand der Wissenschaft gilt. Die berufsspezifischen Sorgfaltspflichen richten sich in erster Linie nach dem medizinischen Standard des jeweiligen Fachgebiets, der unter anderem normiert wird durch entsprechende Leitlinien der berufsständischen Organisationen, z. B. den Landeszahnärztekammern, so etwa in der Notfall-Richtlinie der Landeszahnärztekammer Baden-Württemberg. Darin wird explizit das Vorhandensein einer Notfallausrüstung gefordert, die regelmäßig zu überprüfen ist, insbesondere das Haltbarkeitsdatum der Notfallmedikamente, die nach Ablauf auszutauschen sind. Auch werden regelmäßige Schulungen des gesamten Teams gefordert.

djz: Können aus dem Verhalten des Zahnarztes zivil- und/oder strafrechtliche Ansprüche entstehen?

Wandtke: Der Behandlungsvertrag zwischen Zahnarzt und Patient verpflichtet den Zahnarzt zu besonderer Sorgfalt, deren schuldhafte Verletzung Schadensersatzansprüche des Patienten auslösen kann. Daneben haftet der Zahnarzt wegen eines Behandlungsfehlers oder der Unterlassung einer gebotenen Hilfeleistung auch nach den Vorschriften der unerlaubten Handlung ( $\$ \$ 823 \mathrm{ff} . \mathrm{BGB})$ auf Schadensersatz und Schmerzensgeld. Strafrechtlich beruht die besondere Verantwortlichkeit des Zahnarztes auf der tatsächlichen Übernahme der Behandlung. Der Zahnarzt übernimmt damit eine Garantenstellung, die ihn im Notfall zum Handeln zwingt und verpflichtet, eine durch die Behandlung eingetretene Gefahr von seinem Patienten abzuwenden. Mögliche Komplikationen müssen ebenso bekannt sein wie erforderliche Gegenmaßnahmen. Es liegt also auf der Hand, dass sachdienliche erste Hilfe in bestimmten Fällen auch das Vorhandensein einer entsprechenden Notfallausrüstung voraussetzt. Ebenso liegt auf der Hand, dass das Vorhalten einer Notfallausrüstung wenig Sinn macht, wenn sie im Ernstfall erst einmal zusammengesucht werden müsste und man zudem mit deren Handling nicht vertraut ist. Es ist noch niemand wegen einer falschen Erstversorgung verurteilt worden. Nichts zu tun, das allerdings hätte auf jeden Fall rechtliche Konsequenzen.

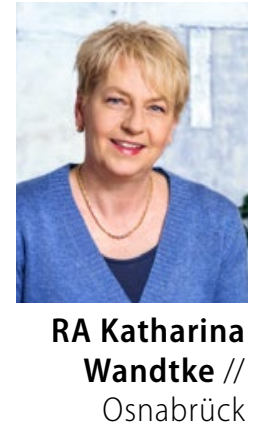

Osnabrück

\section{Hier steht eine Anzeige.}

